\title{
QED-Like Simple High Order Perturbative Relation between the Gravitational Constant $G$ and the Planck Constant $h$
}

\author{
Matt Kalinski \\ Department of Chemistry and Biochemistry, Utah State University, Logan UT, USA \\ Email: matt.kalinski@aggiemail.usu.edu
}

How to cite this paper: Kalinski, M. (2021) QED-Like Simple High Order Perturbative Relation between the Gravitational Constant $G$ and the Planck Constant $h$. Journal of High Energy Physics, Gravitation and Cosmology, 7, 595-601.

https://doi.org/10.4236/jhepgc.2021.72034

Received: January 22, 2021

Accepted: April 19, 2021

Published: April 22, 2021

Copyright $\odot 2021$ by author(s) and Scientific Research Publishing Inc. This work is licensed under the Creative Commons Attribution International License (CC BY 4.0).

http://creativecommons.org/licenses/by/4.0/

\section{(c) (i) Open Access}

\begin{abstract}
We find a simple precise formula for the gravitational constant $G$ relating it to the electron charge, electron mass, the vacuum dielectric constant and the speed of light (or magnetic permeability of the vacuum) in power of the fine structure constant i.e. relating the gravitational constant to the Planck constant through others which also well exist without the quantum mechanics therefore relating two fundamental constants as not independent through the parameters of the electron and the electromagnetic properties of the vacuum.
\end{abstract}

\section{Keywords}

Gravitational Constant, Planck Constant, Quantum Gravity, Theory of Everything, Theory of the Electron Structure

\section{Introduction}

The ratio of the proton to the electron mass was once noticed [1] as magical

$$
\mu=\frac{m_{p}}{m_{e}}=6 \pi^{5}=1836.12
$$

The 1951 ratio can be further adjusted to the most accurate current value by the simple tuning multiplicative factor $\sqrt[36821]{2}$ i.e. 36821 -st root of 2

$$
\mu=6 \pi \sqrt[536821]{2}=1836.152673
$$

We find here a simple precise analytical formula for the gravitational constant $G$ relating it to the electron charge, electron mass, the vacuum dielectric constant and the speed of light (or magnetic permeability of the vacuum) in power of the fine structure constant as it was only a constant of the Quantum Electrodynamics (QED) [2], i.e. relating the gravitational constant to the Planck con- 
stant through others which also well exist without the quantum mechanics therefore relating two fundamental constants as not independent through the parameters of the electron and the electromagnetic properties of the vacuum. We asked for the following conditions: 1) The gravitational constant is proportional to the natural strong gravity [3], gravitational constant for the electron which already can be constructed from the dimensional analysis of units, simply

$$
G_{\mathrm{s} 0}=\frac{\hbar c}{m_{e}^{2}}=3.80994 \times 10^{34} \frac{\mathrm{m}^{3}}{\mathrm{~kg} \cdot \mathrm{s}^{2}}
$$

2) It is proportional as the QED perturbation expansion dimensionless coupling constant i.e. in power of the fine structure constant $\alpha$ so it formally looks like the $n$-th order perturbation correction to the electron-electron Coulomb interaction through the Yukawa exchange mechanism [4] of the logitudinal photons but is insensitive to the sign of charge. 3) The front rest coefficient is around 1.4) In similarity to the proton electron mass ratio, it can be expressed as a simple combination of the integer and the irrational numbers and 5) The analytical formula is perfectly matching the best known experimental CODATA value.

\section{The Preliminary Newtonian Value}

The preliminary formula yields

$$
G=\frac{4}{3} \alpha^{20}\left(\frac{e}{m_{e}}\right)^{2} \frac{1}{4 \pi \varepsilon_{0}}=\frac{4}{3} \alpha^{21} \frac{\hbar c}{m_{e}^{2}}=6.79769 \times 10^{-11} \frac{\mathrm{m}^{3}}{\mathrm{~kg} \cdot \mathrm{s}^{2}}
$$

i.e. gravitational constant is proportional in (4/3) of the 20-th power of the fine structure constant $\alpha=e^{2} /\left(4 \pi \varepsilon_{0} \hbar c\right) \approx 1 / 137$, to the square of the ratio between the electron charge $e$ and its mass $m_{e}$ divided by $4 \pi$ times the vacuum dielectric constant $\varepsilon_{0}$ or in 21-th power of the fine structure constant to the strong gravitational constant defined here as

$$
G_{s}=\frac{4}{3} \frac{\hbar c}{m_{e}^{2}}=5.07992 \times 10^{34} \frac{\mathrm{m}^{3}}{\mathrm{~kg} \cdot \mathrm{s}^{2}},
$$

which shows immediately the electron-electron gravitational interaction as an effective ultra-small electromagnetic (electrostatic) interaction of some order in $\alpha$ and that the gravitational energy corrections to the electron-electron electromagnetic interactions are in the 20-th power of the fine structure constant. The value is that obtained by Newton [5] by the rough estimate of the Earth density as the 6 times that of water. This is in agreement with [6] predicting the approximate (when the complex $i,|i|=1$ is assumed approximately 1 within the $\varepsilon \leq 1 \ll 10$ complex numbers neighborhood circle $100+i$ is approximately 101 in that sense) "Rydberg" spectrum of the elementary particles with masses $m_{n}$ from the formula (34) in [6] as

$$
G=\frac{1}{2 n^{2}} \frac{\hbar c}{m_{n}^{2}}
$$

i.e. electron for 


$$
\begin{gathered}
n \approx\left(\frac{1}{\alpha}\right)^{21 / 2}\left(\frac{3}{8}\right)^{1 / 2} \\
n=16740933742287336243200
\end{gathered}
$$

as a Big Bang ultra-high random "Rydberg toss" excitation which is approximately assuming that the elementary particle is a discharged Black Hole which self binds itself orbiting itself on a circular orbit with the Schwarzschild radius without the relativistic mass gain with the speed of light on its own mass with the fractional closed multiple-spiral near-circular orbit "hydrino" [7] Bohr quantization i.e. is a fractional Planck particle when only the integer fraction of its Compton wavelength is its Schwarzschild circumference ( $2 \pi$ times the Schwarzschield radius) of its mass i.e.

$$
\frac{1}{N} \lambda_{\mathrm{NC}}=\frac{1}{N} \frac{h}{m_{N} c}=2 \pi r_{s}=2 \pi \times \frac{2 G m_{N}}{c^{2}}
$$

or

$$
G=\frac{1}{2 N} \frac{\hbar c}{m_{n}^{2}}
$$

or

$$
N=n^{2} .
$$

It also agrees up to the small multiplicative factor $2 \sqrt{2}$ with self-consistently solving the exact nonrelativistic Schrödinger equation for the Black Hole to obtain its possible quantum mass spectrum [8] treated as the self-bound Hydrogen atom while equalizing its Rydberg energy to the rest relativistic energy of the minus half of its effective mass but considering also the "hydrino" fractional quantum numbers i.e. $N \rightarrow 1 / N$.

The leading order of the Lamb shift [9] is in the 6-th power of $\alpha$ in that sense. The formula immediately predicts the Positronium ground state [10] gravitational shift (and so the between 1 - 2 levels gap gravitational "Lamb shift" as $-3 / 4$ of it) by the effective attractive Coulomb coupling renormalization $e^{2} \rightarrow e^{2}\left(1+(4 / 3) \alpha^{20}\right)$ entering the Rydberg energy $R_{\infty}$ in square in the leading order as

$$
\delta E_{g}=-2 \times 6.8 \times \frac{4}{3} \alpha^{20} \mathrm{eV}=-3.3 \times 10^{-42} \mathrm{eV}
$$

and so $2 \times 6 \times \pi^{5}$ of that for the Hydrogen and twice of that for the Deuterium ground state respectively. Assuming the somehow perfectly Mössbauer-rigid nucleus in both cases not to change the Kepler reduced mass and eliminate the native isotope effect it leads mathematically to the Trojan or anti-Trojan Wave Packet generation [11] from the same circular quantum number state in atom Rydberg state of two different isotopes due to the electron-nucleus gravity as big as of $10^{68}$ meters radius ( $10^{42}$ times the size of the Universe) i.e. with

$$
n=\frac{R_{\infty}}{\delta R_{\infty}},
$$


$n=10^{39}$. It also takes about $10^{27}$ seconds ( $10^{9}$ ages of the Universe) (and so only $10^{-27}$ of it within one second) to cause one interference fringe shift assuming two counter rotating Trojan Wave Packets around the principal quantum number $n=10$ which belong to two Hydrogen atom of two different isotopes can be interfered between each other with the method of delta pulse ionization described in [12].

\section{The Refined Value}

The simple correcting prefactor $1 / \sqrt[38]{2}$ (inverse of the 38-th root of 2 ) is further adjusting the value to the best known experimental [13] CODATA value giving

$$
\begin{aligned}
G & =\frac{1}{\sqrt[38]{2}} \frac{4}{3} \alpha^{21} \frac{\hbar c}{m_{e}^{2}}=6.674330065689801 \times 10^{-11} \mathrm{~m}^{3} /\left(\mathrm{kg} \cdot \mathrm{s}^{2}\right) \\
& \approx(130923 / 100000) \alpha^{21} \frac{\hbar c}{m_{e}^{2}} .
\end{aligned}
$$

It may be interpreted as a ratio factor between the gravitational self energy radius $r_{g}$ and the electromagnetic electron radius $r_{e}$ when the gravitational self energy deficiency in modulus is assumed to be the $(4 / 3) \alpha^{20}$ quantum fraction of the electrostatic one i.e.

$$
\begin{gathered}
E_{g}=-\frac{G m_{e}^{2}}{r_{g}}=-\frac{4}{3} \alpha^{20} E_{e} \\
E_{e}=m_{e} c^{2}=\frac{1}{4 \pi \varepsilon_{0}} \frac{e^{2}}{r_{e}}
\end{gathered}
$$

i.e. $r_{e}=\sqrt[38]{2} r_{g}$. This is in inequality $r_{e}>r_{g}\left(r_{e}=r_{g} \sqrt[8]{2}\right)$ agreement with the classical model of the electron with the spin $\hbar / 2$ assuming slightly different rotating Gaussian mass and charge distributions inside the electron

$$
\begin{gathered}
\rho_{e}(r)=e \mathcal{N} e^{-r^{2} / r_{e}^{2}} \\
\rho_{m}(r)=m_{e} \mathcal{N} e^{-r^{2} / r_{g}^{2}}
\end{gathered}
$$

predicting the anomalous electron $g$-factor 2 if only

$$
\left(\frac{r_{e}}{r_{m}}\right)^{8}=2,
$$

as it was built from the solid electrified matter but with the locally variable density and non-proportional variable level of electrification. The refined "Rydberg" quantum number of the electron as the excitation itself including the correction is now $n=16894315429949215866880$ (Table 1).

We so find (Figure 1)

$$
G=\frac{4}{3 \sqrt[38]{2}} \frac{\hbar c}{m_{e}^{2}} \alpha^{21}=1.30923 \times \frac{\hbar c}{m_{e}^{2}} \alpha^{21}=6.67433 \times 10^{-11} \frac{\mathrm{m}^{3}}{\mathrm{~kg} \cdot \mathrm{s}^{2}},
$$

where $G$ is the gravitational constant,

$$
\hbar=1.05457181710^{-34} \mathrm{~J} \cdot \mathrm{s}
$$




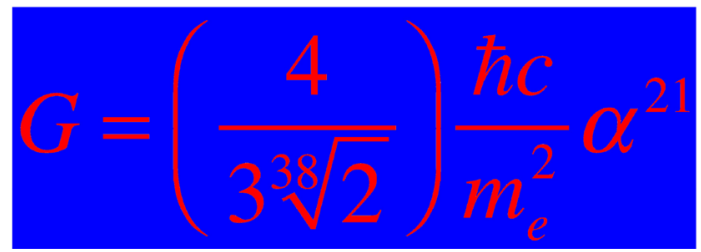

Figure 1. The picture of the discovered "Theory of Everything" formula expressing the gravitational constant $G$ in terms of the other fundamental constants and the electron parameters as the elementary particle (charge and mass).

Table 1. The table showing the progressive refinement of the value of the strong gravity constant $G_{s}$ and the gravitation constant $G$ in terms of other fundamental constants.

\begin{tabular}{|c|c|c|c|}
\hline Level & $G_{s}$ formula & $G_{s}$ value $\left(\mathrm{m}^{3} \cdot \mathrm{kg}^{-1} \cdot \mathrm{s}^{-2}\right)$ & $G=G_{s} \alpha^{21}$ value $\left(\mathrm{m}^{3} \cdot \mathrm{kg}^{-1} \cdot \mathrm{s}^{-2}\right)$ \\
\hline (1) & $\frac{\hbar c}{m_{e}^{2}}$ & $3.80994 \times 10^{34}$ & $5.09789 \times 10^{-11}$ \\
\hline (2) & $\frac{4}{3} \frac{\hbar c}{m_{e}^{2}}$ & $5.07992 \times 10^{34}$ & $6.79769 \times 10^{-11}$ \\
\hline (3) & $\frac{4}{3 \sqrt[38]{2}} \frac{\hbar c}{m_{e}^{2}}$ & $4.98811 \times 10^{34}$ & $6.67433 \times 10^{-11}$ \\
\hline (4) & $\frac{4}{3 \sqrt[38]{2} \sqrt[160000]{2}} \frac{\hbar c}{m_{e}^{2}}$ & $4.98809 \times 10^{34}$ & $6.67430 \times 10^{-11}$ \\
\hline
\end{tabular}

$$
\begin{gathered}
c=299792458 \frac{\mathrm{m}}{\mathrm{s}} \\
m_{e}=9.1093837015 \times 10^{-31} \mathrm{~kg} \\
\alpha=1 / 137.035999206=7.2973525693 \times 10^{-3},
\end{gathered}
$$

$\hbar$ is the reduced Planck constant, $c$ is the speed of light, $m_{e}$ is the electron mass and $\alpha$ is the fine structure constant i.e. with the perfect agreement with the most recent CODATA [13] value only if the right side quantities are also the CODATA values. This expresses explicitly that the gravitational interaction between the electrons may be considered as an exotic ultra-weak Coulomb attractive electromagnetic (electrostatic) interaction of the 20-th order in the fine structure constant $\alpha$ and therefore is normally neglected in QED as much below the highest 7-th order in $\alpha$ normally considered there. It also defines electron strong gravity constant as

$$
G_{s}=\frac{4}{3 \sqrt[38]{2}} \frac{\hbar c}{m_{e}^{2}}=4.98811 \times 10^{34} \frac{\mathrm{m}^{3}}{\mathrm{~kg} \cdot \mathrm{s}^{2}} .
$$

The further ultra-fine tuning prefactor placing the value perfectly at the center of the CODATA error field can be found as $1 / \sqrt[160000]{2}$ (hundred sixty thousandth root of 2) i.e.

$$
G=\frac{4}{3 \sqrt[38]{2} \sqrt[160000]{2}} \frac{\hbar c}{m_{e}^{2}} \alpha^{21}=1.30923 \times \frac{\hbar c}{m_{e}^{2}} \alpha^{21}=6.67430 \times 10^{-11} \frac{\mathrm{m}^{3}}{\mathrm{~kg} \cdot \mathrm{s}^{2}},
$$

with the method of power 2 approximants of the number which is close to 1 as 
for example for $\pi / 3$

$$
\begin{gathered}
\pi=3.141592653589793 \\
\pi \approx 3 \sqrt[35]{2} / \sqrt[7509]{2} \cdots=3.1415923582882304
\end{gathered}
$$

etc. for which the ultra-refined

$$
G_{s}=\frac{4}{3 \sqrt[38]{2} \sqrt[160000]{2}} \frac{\hbar c}{m_{e}^{2}} \alpha^{21}=4.98809 \times 10^{34} \frac{\mathrm{m}^{3}}{\mathrm{~kg} \cdot \mathrm{s}^{2}} .
$$

\section{Summary}

We find here the "Theory of Everything" formula relating the gravitational constant to the Planck constant through the electron mass, speed of light and the fine structure constant (electric charge and the dielectric permittivity of the vacuum) suggesting that the electron quantum mechanics is a result of the gravitational and dielectric properties of the vacuum or alternatively the gravity of electrons is an ultra-weak QED effect of longitudinal virtual quantum photons interacting with the 1-st scalar component of the electron 4-current and the Yukawa-like interaction between charges, which is insensitive to the sign of the charge. The $4 / 3$ and $1 / 2^{1 / 38}$ ( 1 over 38 -th root of 2 ) coefficients adjusting the 21-st $\alpha$ power order of the so defined strong gravity constant $G_{s 0}=\hbar c / m_{e}^{2}$ to the first Newtonian 15-teen century value and than the perfect CODATA value are easy to remember as having the $4 / 3$ factor of the sphere volume and $1 / 38$ in the 2 exponent as the calculator LCD display altered value of the approximate fine structure constant $1 / 137$. Yet another interpretation is that the parameters of the electron as the elementary particle like the mass $m$ and charge $e$ are fully determined by the properties of the vacuum and the quantum mechanics i.e. the value of $\hbar$ by some Planck particle like mechanism of de Broglie standing wave Black Hole self no-escape for example with fractional angular momentum. Perfect CODATA values of all involved quantities must be used for the G CODATA value agreement.

\section{Acknowledgements}

Author would like to thank W. Tarkowski, the author of [6] for the valuable discussion regarding his paper used for the partial explanation of the discovered formula formulating the model of the elementary Planck-like particle as the self-bound Black-Hole in the extended 5-dimensional space-time.

\section{Conflicts of Interest}

The author declares no conflicts of interest regarding the publication of this paper.

\section{References}

[1] Lentz, F. (1951) The Ratio of Proton and Electron Masses. Physical Review, 82, 554. https://doi.org/10.1103/PhysRev.82.554.2 
[2] Mandl, F. (1959) Introduction to Quantum Field Theory. Interscience Publishers Inc., Hoboken.

[3] Salam, A. (1993) Strong Gravity Approach to QCD and Confinement. Modern Physics Letters A, 8, 321-326. https://doi.org/10.1142/S0217732393000325

[4] Yukawa, H. (1935) On the Interaction of Elementary Particles. Proceedings of the Physico-Mathematical Society of Japan, 17, 48-57.

[5] Newton, I. (1687) Philosophiæ Naturalis Principia Mathematica, London. https://doi.org/10.5479/sil.52126.39088015628399

[6] Tarkowski, W. (2004) A Toy Model of the Five-Dimensional Universe with the Cosmological Constant. International Journal of Modern Physics A, 19, 5151-5084. https://doi.org/10.1142/S0217751X04019366

[7] Mills, R.L. (2020) The Grand Unified Theory of Classical Physics. Vol. I, Atomic Physics, Library of Congress Control Number 2015915593.

[8] Corda, C. (2020) On Black Hole Schrödinger Equation and Gravitational Fine Structure Constant. arXiv:2012.08967

[9] Lamb, E.W. and Retherford, R.C. (1947) Fine Structure of the Hydrogen Atom by a Microwave Method. Physical Review, 72, 241-243.

https://doi.org/10.1103/PhysRev.72.241

[10] Eides, M.I. and Grotch, H. (1995) Corrections of Order $\alpha^{6}$ to S Levels of Two-Body Systems. Physical Review, 52, 1757-1760. https://doi.org/10.1103/PhysRevA.52.1757

[11] Kalinski, M. and Eberly, J.H. (1998) New States of Hydrogen in a Circularly Polarized Electromagnetic Field. Physical Review A, 77, 2420-2423.

https://doi.org/10.1103/PhysRevLett.77.2420

[12] Kalinski, M. (1998) Aharonov-Bohm Oscillations in a Hydrogen Atom in a Radiation Field through Electron Self-Interference. Physical Review A, 57, 2239-2242. https://doi.org/10.1103/PhysRevA.57.2239

[13] 2018 CODATA Value: Newtonian Constant of Gravitation. 2019 The NIST Reference on Constants, Units, and Uncertainty NIST. 